\title{
Robust Partitioned Scheduling for Real-Time Multiprocessor Systems
}

\author{
Frédéric Fauberteau ${ }^{1}$, Serge Midonnet $^{1}$, and Laurent George ${ }^{2}$ \\ 1 Université Paris-Est, \\ LIGM, UMR CNRS 8049, 5, bd Descartes, \\ Champs-sur-Marne, 77454 Marne-la-Vallée CEDEX 2, France \\ \{fauberte, midonnet\}@univ-mlv.fr \\ 2 ECE / LACSC, \\ 37, quai de Grenelle, 75015 Paris, France \\ lgeorge@ieee.org
}

\begin{abstract}
In this paper, we consider the problem of fixed-priority partitioned scheduling of sporadic real-time tasks for homogeneous processors. We propose a partitioning heuristic that takes into account possible Worst Case Execution Time (WCET) overruns. Our goal is to maximize the duration a task can be allowed to exceed its WCET without compromising the timeliness constraints of all the tasks. This duration is denoted in the paper the allowance of the task and is computed with a sensitivity analysis. The partitioning heuristic we propose, assigns the tasks to the processors in order (i) to maximize the allowance of the tasks and (ii) to tolerate bounded execution duration overruns. Property (ii) is important as real-time applications are often prone to be subject to OS approximations or software faults that might result in execution duration overruns. We show with performance evaluations that Allowance-Fit-Decreasing partitioning improves the temporal robustness of real-time systems w.r.t. classical $\{$ First-Fit/Best-Fit/Next-Fit\}-Decreasing partitioning.
\end{abstract}

Keywords: Real-time Scheduling, Partitioned Scheduling, Robustness.

\section{Introduction}

Fixed-priority scheduling of recurring real-time tasks has been largely studied for uniprocessors. In such a scheduling, a Priority Assignment (PA) assigns a fixed priority to each job of the task. For instance, Rate-Monotonic (RM) is an optimal PA for periodic tasks with implicit-deadlines (deadlines equal to periods) 1]. Optimality implies that if a feasible PA over a taskset exists, then the optimal $\mathrm{PA}$ is also feasible. A feasible taskset is a taskset such that a scheduling algorithm exists which can schedule this taskset. We focus on the more general model of tasks with constrained-deadlines (deadlines less than or equal to periods) for which Deadline-Monotonic (DM) is an optimal PA 2]. Recently, the optimal Robust Priority Assignment (RPA) [3] has been proposed to find the PA which maximizes the interference that a tasks system can support. These interferences 
can be handled by the tasks by allowing WCETs overruns while the timeliness constraints of all the tasks are respected. These tolerated WCETs overruns are denoted allowance of the tasks. In the same way, our motivation is to propose a robust multiprocessor scheduling which maximizes the allowance.

The two most studied approaches to schedule real-time tasks on a multiprocessor are partitioned and global scheduling. The first one does not allow tasks to migrate whereas the second one allows unrestricted migrations. Recent architecture have reduced the cost of migration. Nevertheless, taking into account the cost of migration in the feasibility conditions of global scheduling is still an open issue. A recent performance evaluation of partitioned and global schedulings show that partitioned scheduling outperform global scheduling, in the current state-of-the-art of feasibility conditions [4. We therefore focus on the partitioned approach. Several algorithms for fixed-priority partitioned scheduling have been proposed [56/7/89]. The aim of the authors is to propose algorithms which improve the worst-case utilization bound. The worst-case utilization bound for a scheduling algorithm $A$ is defined as the minimum utilization for which any implicit-deadline taskset is schedulable according to algorithm $A$. The utilization of an implicit-deadline taskset is the sum of the processor utilization (formally defined in Sect 2) of each task composing this taskset.

In this paper, our motivation is slightly different since we want to design a partitioned scheduling which improves the temporal robustness of a system i.e. to improve its capability to support variations on the system parameters at run time (WCET overruns for e.g.). Such events should not lead to a deadline violation in a hard real-time application. We focus on the WCET parameter and we propose an algorithm which allocates the tasks on the processor having the greatest capability to support WCETs overrun by maximizing the minimum allowance of all the tasks.

The rest of this paper is organized as follows. In Section 2, we introduce the terminology used in the rest of this paper. In Section 3 we give a definition of robustness in context of this paper. In Section 4, we discuss two manners to compute the allowance of the execution duration which is the criterion of our partitioning algorithm for the assignment of real-time tasks on the processors. In Section 5, we present our heuristic and describe how it works. In Section 6] we compare on some simulations the performance of the partitioning schedulings and we explain the benefits of our approach. We summarize the contributions of this paper in Section 7 and we give direction for our future work.

\section{Terminology}

In this paper, we consider an application built from a set $\tau=\left\{\tau_{1}, \tau_{2}, \ldots, \tau_{n}\right\}$ of $n$ sporadic real-time tasks. A sporadic task is a recurring task for which only a upper bound on the separation between release times of the jobs is known. Each task $\tau_{i}$ is characterized by a minimum interarrival time $T_{i}$ (also denoted period), a worst-case execution time $C_{i}$ and a relative deadline $D_{i}$. This application runs on a platform $\Pi=\left\{\pi_{1}, \pi_{2}, \ldots, \pi_{m}\right\}$ of $m$ identical processors (homogeneous 
case). We consider a fixed-priority scheduling on each processor. A fixed-priority scheduler assigns a priority to each task and all jobs of a task is released with the fixed priority of this task. We assume that tasks are indexed by decreasing priority: $\forall i=1, \ldots, n-1$, task $\tau_{i}$ has a higher priority than task $\tau_{i+1}$. A partitioning algorithm produces a partition $\operatorname{Part}(\tau)=\left\{\tau^{1}, \tau^{2}, \ldots, \tau^{m}\right\}$ of $m$ disjointed subsets where each subset $\tau^{j}$ of real-time tasks is executed on processor $\pi_{j}$. The subset $\tau^{j}$ composed by $n_{j}$ tasks is also denoted by $\tau^{j}=\left\{\tau_{1}^{j}, \tau_{2}^{j}, \ldots, \tau_{n_{j}}^{j}\right\}$. In the rest of this paper, we refer to $\tau_{i}$ when the considered task is taken independently and to $\tau_{i}^{j}$ when it is considered assigned on processor $\pi^{j}$. We define $u_{i}$ as the utilization of task $\tau_{i}: u_{i} \equiv \frac{C_{i}}{T_{i}}$ and $U^{j}$ as the utilization of the taskset $\tau^{j}$ : $U^{j} \equiv \sum_{\tau_{k}^{j} \in \tau^{j}} u_{k}$. On the processor $\pi_{j}$, we denote $l p^{j}(i)$ (respectively $h p^{j}(i)$ ) the subset of real-time tasks assigned to $\pi^{j}$ which have a priority lower than (respectively higher than or equal to) $\tau_{i}$. The response time of the task $\tau_{i}$ is denoted $R_{i}$. We denote $R_{i}^{k}$ the $k^{t h}$ iteration in the response time computation of the task $\tau_{i}$.

\section{Robustness}

We consider the robustness in the real-time systems as the capacity of the system to handle WCET overruns faults when the WCET are estimated. If the WCET of all the tasks of the system has been well defined, a feasibility analysis shows wheter the system is feasible. But in pratice, it may possible that a task makes a fault or that the time constraints has been miscalculated. Some real-time specifications - such as Real-Time Specification for Java [10] - provide mechanism to handle cost overruns and deadline misses in the case of estimated WCET.

In this work, we consider the robustness as the capacity of a system to meet all the deadlines. We can guarantee that the system stay feasible if and only if we know the execution duration during which a task can exceed its WCET without any deadline is missed. This duration is denoted allowance and the more each task allowance has, the more robust the system regarding to our definition is.

\section{Allowance Concept}

The allowance of a task is used as a criterion for allocating a task on a processor by our heuristic. We define the allowance $A_{i}^{j}$ of a task $\tau_{i}^{j}$ on the processor $\pi^{j}$ as follows :

Definition 1. Let $\tau^{j}$ be a given set of tasks assigned on processor $\pi^{j}$. The allowance $A_{i}^{j}$ of a task $\tau_{i}^{j}$ of $\tau^{j}$ is the maximum duration which can be added to the WCET $C_{i}$ of $\tau_{i}^{j}$ such as all tasks of $\tau^{j}$ meet their deadlines.

We identified in the literature two approaches to compute the allowance: one based on a (Worst Case Response Time) WCRT computation and one based on a sensitivity analysis on the WCETs. The response time of a task is the duration between the time this task has been released and the time it has been 
Table 1. System of 4 sporadic real-time tasks

\begin{tabular}{c|ccc}
\hline & $C_{i}$ & $D_{i}$ & $T_{i}$ \\
\hline$\tau_{1}$ & 10 & 60 & 70 \\
$\tau_{2}$ & 15 & 85 & 100 \\
$\tau_{3}$ & 30 & 190 & 210 \\
$\tau_{4}$ & 45 & 260 & 320 \\
\hline
\end{tabular}

finished. The WCRT of a task is the response time of this task in the worst activation scheme. We use the taskset given in Tab1 to describe the two allowance computation methods in the following subsections.

\subsection{Allowance Computed from WCRT}

One of the available approach to compute the allowance of the execution duration has been proposed by Bougueroua et al. [11. For a given value of allowance $A_{i}^{j}$, this method consists in checking that the system remains schedulable when the execution duration of a task $\tau_{i}$ is equal to $C_{i}^{\prime}=C_{i}+A_{i}^{j}$. In other words, this method consists in checking that the WCRT of all the tasks remains less than or equal to their deadline when their WCET is extended to $C_{i}+A_{i}^{j}$. The 3 following equations perform this check for the task $\tau_{i}^{j}$ on a processor $\pi_{j}$ if $\tau_{i}^{j}$ was assigned on $\tau^{j}$.

$$
\begin{aligned}
& U^{j}+\frac{A_{i}^{j}}{T_{i}} \leq 1 \\
& R_{i}^{k+1}=C_{i}+A_{i}^{j}+\sum_{\tau_{h} \in h p^{j}(i)}\left\lceil\frac{R_{i}^{k}}{T_{h}}\right\rceil C_{h} \leq D_{i} \\
& \forall \tau_{l} \in l p^{j}(i), \\
& R_{l}^{k+1}=C_{l}+\sum_{\tau_{h} \in h p^{j}(l)}\left\lceil\frac{R_{l}^{k}}{T_{h}}\right\rceil C_{h}+\left\lceil\frac{R_{l}^{k}}{T_{i}}\right\rceil A_{i}^{j} \leq D_{l}
\end{aligned}
$$

The value of allowance of a real-time task $\tau_{i}$ is found by a binary search. Equation (11) tests if the utilization $U^{j}$ of the system when the WCET of $\tau_{i}^{j}$ is extended to $C_{i}+A_{i}^{j}$ does not exceed processor utilization. An upper bound $A_{i, u p}^{j}$ on the allowance of the task $\tau_{i}$ can be found from (1):

$$
A_{i, u p}^{j}=\left\lfloor\left(1-U^{j}\right) \cdot T_{i}\right\rfloor
$$

Equation (4) allows to bound the binary search in $\left[0, A_{i, u p}^{j}\right]$. For the task $\tau_{1}^{j}$ of our example, $A_{1, u p}^{j}=\lfloor(1-0.58) \cdot 70\rfloor=29$. We can carry out a binary search with $0 \leq A_{1} \leq 29$. Equation (2) tests if the response time $R_{1}$ of $\tau_{1}$, when its WCET has been extended to $C_{1}+A_{1, j}$, does not exceed its deadline $D_{1}$. Equation (3) tests if the response times $R_{l}$ of all tasks $\tau_{l}^{j}$ of lower priority 
than $\tau_{1}^{j}$ don't exceed their deadlines $D_{l}$ when the WCET of $\tau_{1}^{j}$ is extended by $A_{1, j}$. For the value $\lfloor 29 / 2\rfloor=14$, we must check that this value satisfies (2) and (3). For $A_{1, j}=14$, the response time of $\tau_{1}^{j}$ is $R_{1}=24 \leq D_{1}$. We also obtain $R_{2}=39 \leq D_{2}, R_{3}=69 \leq D_{3}$ and $R_{4}=177 \leq D_{4}$. Then $A_{1, j}=14$ is a valid value of allowance for $\tau_{1}^{j}$ on the processor $\pi^{j}$. We continue the binary search until $A_{1, j}=21$, then $A_{1}=21$ is the maximum value of allowance for $\tau_{1, j}$.

The complexity of this approach is pseudo-polynomial due to the Response Time Analysis (RTA) in (2) and in (3). This complexity is in $O\left(n^{2}\right)$ where $n$ is the number of tasks. Indeed, for a task $\tau_{i}$, a RTA is performed in $O(n)$ and for each task of lower priority than $\tau_{i}$, a RTA is performed in $O(n)$. In the worst case, there is $n-1$ tasks of lower priority than $\tau_{1}$, thus the complexity is $O\left(n^{2}\right)$.

\subsection{Allowance Computed from Sensitivity Analysis}

Another approach to compute the allowance of the execution duration is the sensitivity analysis. This approach has been introduced by Bini et al. 12. This approach is attractive compared to the previous one because no iterative computation (such as WCRT computation) is needed. The authors propose to consider the system only at time corresponding to the activation time of the highest priority tasks in $\left[0, D_{i}\right]$ union time $\left\{D_{i}\right\}$. The maximum allowance $A_{i}^{j}$ of a task $\tau_{i}^{j}$ on the processor $\pi^{j}$ is computed by the following equations:

$$
\begin{aligned}
\operatorname{Sens}(k) & =\max _{t \in \operatorname{sched} P_{k}} \frac{t-\left(C_{k}+\sum_{h \in h p(k)}\left\lceil\frac{t}{T_{h}}\right\rceil C_{h}\right)}{\left\lceil t / T_{i}\right\rceil} \\
A_{i}^{j} & =\left\lfloor\min _{k \in l p(i)} \operatorname{Sens}(k)\right\rfloor
\end{aligned}
$$

where $s c h e d P_{k}$ is the set of scheduling points defined by $\operatorname{sched} P_{k}=\mathcal{P}_{i-1}\left(D_{k}\right)$ and $\mathcal{P}_{k}(t)$ is defined by :

$$
\left\{\begin{array}{l}
\mathcal{P}_{0}(t)=\{t\} \\
\mathcal{P}_{k}(t)=\mathcal{P}_{k-1}\left(\left\lfloor\frac{t}{T_{k}}\right\rfloor T_{k}\right) \cup \mathcal{P}_{k-1}(t)
\end{array}\right.
$$

For the task $\tau_{1}$ of our example, sched $P_{1}=D_{1}=\{60\}$. For the other tasks in the same way, sched $P_{2}=\{70,85\}$, sched $P_{3}=\{70,100,140,190\}$ and sched $P_{4}=$ $\{140,200,210,260\}$. The values $\operatorname{Sens}(1)=50, \operatorname{Sens}(2)=45, \operatorname{Sens}(3)=33.33$ and Sens $(4)=21.66$ are computed by using Equation 5 . The allowance $M_{1}=$ $\min _{k \in l p^{j}(i)}\lfloor\operatorname{Sens}(k)\rfloor=21$ is obtained from (5) and (6) .

The complexity of this approach is exponential because $\left|s c h e d P_{n}\right|$, denoted as the size of the scheduling points set computed by (7) is $2^{n-1}$ in the worst-case. But in practice, the size of $\operatorname{sched} P_{n}<<2^{n-1}$ for a great value of $n$. We notice that the size of $s c h e d P_{n}$ is highly sensitive to the range of task periods as seen in appendix of 13 . 


\section{Partitioning Algorithm}

\subsection{Task Partitioning Problem}

The task partitioning problem consists of finding a partition of a taskset $\tau$ in $m$ subsets $\tau^{j}, 1 \leq j \leq m$ such that each subset is feasible on processor $\pi_{j}$. Since it has been proved that BIN-PACKING problem (NP-hard in the strong sense) can be reduced in polynomial-time to a task partitioning problem [14, no optimal algorithm exists to decide in polynomial-time if a given taskset is feasible. Fortunately, approximation algorithms and heuristics exist to find solutions for the task partitioning problem in polynomial-time. Heuristics for the tasks partitioning problem exist and are versions of the heuristics proposed for the bin-packing problem. The more cited in the literature are First-Fit [8] (FF), Best-Fit [6] (BF) and Next-Fit (NF) [7. These heuristics have been initially designed to minimize the number of bins (respectively the number of processors) for the BIN-PACKING problem (respectively the tasks partitioning problem). Another heuristic Worst-Fit [15], is rarely used because it provides poor performance to solve the BIN-PACKING problem. On the problem of task partitioning, this heuristic allocates tasks to processors where utilization is the lowest. This approach is relevant because we want the best allocation of tasks to maximize the allowance of tasks. We propose in the next subsection a heuristic which allocates tasks to processors that has the greatest allowance rather than processors that has the lowest utilization.

\subsection{Allowance-Fit-Decreasing}

We propose a heuristic, denoted Allowance-Fit-Decreasing, to solve the task partitioning problem. We want to tolerate bounded WCET overruns, a property not considered in classical heuristics. WCETs overruns can be due to OS approximations, faults of the task or WCET under-estimation. By definition, the allowance of a processor is the minimum allowance for all task allocated to the processor. Our goal is to propose a partitioning scheme that assigns a task to the processor whose allowance is maximum.

We describe the Allowance-Fit-Decreasing heuristic with the pseudo-code given in Alg 1. The tasks are first sorted according to their utilization by function sort_task_by_decreasing_utilization() (line 1). For each task $\tau_{i}$ of the taskset $\tau$ (iteration loop at lines 2-17), the proc parameter, denoting the processor on which $\tau_{i}$ is allocated (at line 3 ), is initialized with a null value. The minimum value of $a l$ lowance for the entire system (variable $A_{\min }$ at line 4 ) is first initialized to minus infinity. We then consider all processors in $\Pi$ and find the processor that maximizes the processor allowance. For each processor $\pi_{j}$ (iteration loop at lines 5-11), our heuristic finds the minimum value of processor allowance $A_{\min }^{j}$ computed by function compute_allowance $\left(\pi^{j}, \tau_{i}^{j}\right)$ when $\tau_{i}$ is allocated to $\pi_{j}$ with the method described in Sect 4.2. If after the iteration loop, $A_{\min }$ is greater than or equal to 0 then, $\tau_{i}$ can be assigned to a processor, the one that maximizes the processor allowance, by construction. We then proceed with the other tasks 


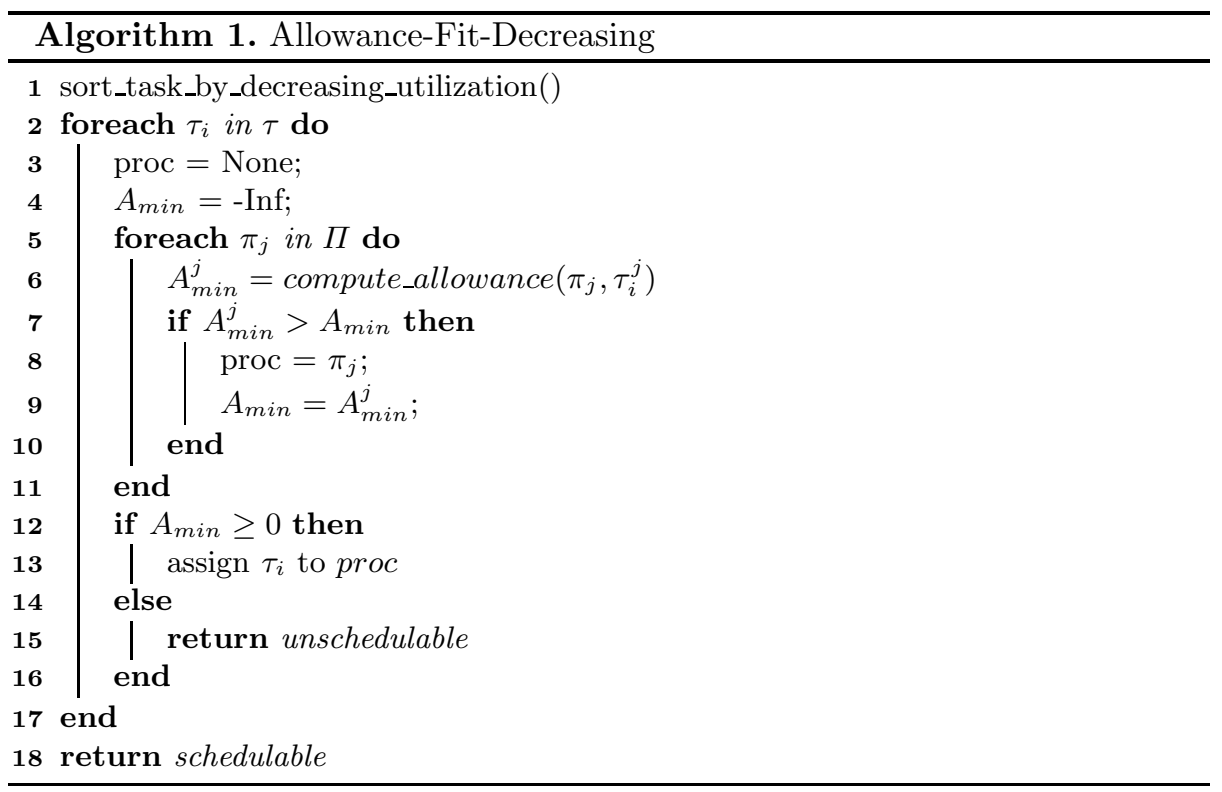

until all the tasks until either all tasks have been assigned to a processor (we then return that the task set is schedulable in line 18) or one task is declared not schedulable (line 15).

\subsection{Partitioned Scheduling Algorithm}

A partitioned scheduling algorithm is the combination of a task partitioning algorithm with a schedulability condition. We build two partitioned scheduling algorithms, one from Worst-Fit and one from Allowance-Fit-Decreasing. For the first one, the schedulability condition is a necessary and sufficient condition implicitly given by Allowance-Fit-Decreasing. Indeed, our heuristic computes with the function compute_allowance $\left(\pi_{j}, \tau_{i}\right)$ the value of $A_{m i n}^{j}$, the minimum allowance for all the tasks assigned to processor $\pi_{j}$, including $\tau_{i}$. If this function returns a negative value, then $\tau_{i}$ cannot be assigned on the processor $\pi^{j}$. $A_{\text {min }}^{j}$ is computed from the sensitivity analysis given in Sect 4.2 . For the second one, we combine Worst-Fit with the necessary and sufficient schedulability condition RTA [16].

During the allocation, the tasks are taken in order of their decreasing utilization. In other words, the tasks with the greater utilization are allocated first. We consider a fixed-priority assignment and we use DM priority assignment since this PA is an optimal one when the considered tasks have constrained-deadlines $\left(\forall i, D_{i} \leq T_{i}\right)[2]$. 


\section{Simulations}

\subsection{Methodology}

Our simulations is based on randomly generated tasksets. Because we focus on tasks with constrained-deadlines, we consider tasksets such that for any task $\tau_{i}, \alpha=\frac{D_{i}}{T_{i}}$. We randomly generate 10 sets for $\alpha \in[0.1,0.2, \ldots, 1.0]$. Each set is built from 100,000 randomly generated tasksets. Each taskset is composed by 24 tasks. A taskset is generated using the UUniFast algorithm [17] which produces a uniformly distributed set of task utilizations and which avoids bias in the generated tasksets. For a given task $\tau_{i}$, the period $T_{i}$ is generated with a uniform distribution between 100 and $100,000 \mathrm{~ms}$. The deadline $D_{i}$ is given by $D_{i}=\alpha \cdot T_{i}$ and the WCET $C_{i}$ is given by $C_{i}=u_{i} \cdot T_{i}$. We consider an homogeneous processor composed by 8 identical processors.

\subsection{Simulation Results}

We show in Fig 1 the average number of iterations during the computation of allowance.

$$
R_{i}^{k+1}=C_{i}+\sum_{\tau_{h} \in h p^{j}(i)}\left\lceil\frac{R_{i}^{k}}{T_{h}}\right\rceil C_{h}
$$

We implement a function iteration() which computes the value given by (8). The computation of the allowance based on the WCRT computation calls this function in (2) and in (3). The computation of the allowance based on the sensitivity analysis calls this function in (5). For the two implementations of the allowance computation, we count the number of calls to the function iteration() for each randomly generated taskset and we keep the average of the allowance over all the taskset. We notice that despite the fact of the complexity of the sensitivity analysis seems greater than the complexity of the allowance computation based on the WCRT, the number of iterations for sensitivity analysis is well below the number of iterations for computation based on the WCRT. We therefore choose to compute allowance by sensitivity analysis [12.

We show in Fig,2, 3and 5 the comparison between First-Fit-Decreasing (FFD), Worst-Fit-Decreasing (WFD) and Allowance-Fit-Decreasing (AFD). Decreasing means that these heuristics assign the tasks in order of their decreasing utilization. We voluntarily omit to show results concerning $\mathrm{BF}$ and $\mathrm{NF}$ because their behavior are very similar to FF. AFD and WFD use a necessary and sufficient condition of schedulability. Therefore we used the necessary and sufficient condition RTA [16, for the heuristics FFD.

We show in Fig 2 the number of partition found by the heuristics FFD, WFD and AFD. We notice that FFD provides a slightly better schedulability for $\alpha \geq$ 0.4. This result is explained by the fact that FFD is one of the best heuristic for the task partitioning problem in terms schedulability. But the gap between FFD and the other two heuristics is not very large and may be acceptable if we want more robustness to WCETs overruns. 


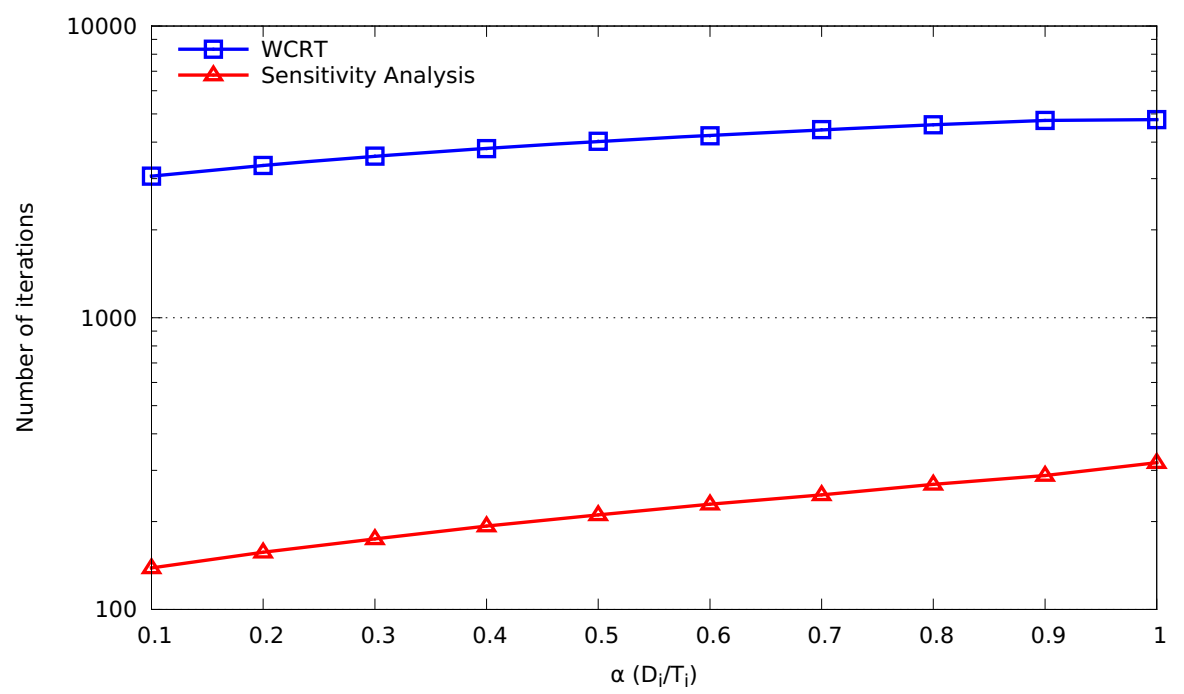

Fig. 1. Comparison of computation time for the allowance computation approaches

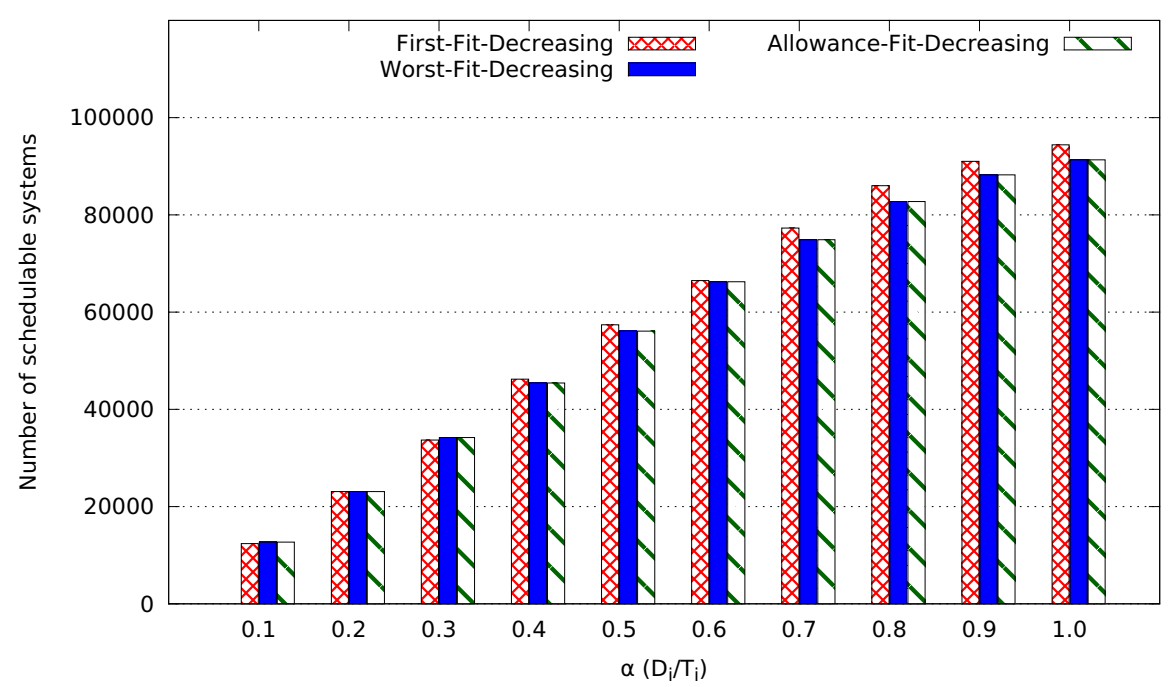

Fig. 2. Comparison of schedulability for the tasks partitioning heuristics

We compare in Fig 3 the minimum allowance obtained by the three heuristics on 4 processors and in Fig 4 on 8 processors. Minimum allowance $A_{\text {min }}$ guarantees that any task of the system can bear an interference during $A_{\text {min }}$ without any deadline is missed. We show that AFD and WFD outperforms largely FFD. Indeed, AFD and WFD distributes the tasks among the processors instead of fills up all the first processors. We note that AFD is slightly better than WFD. 


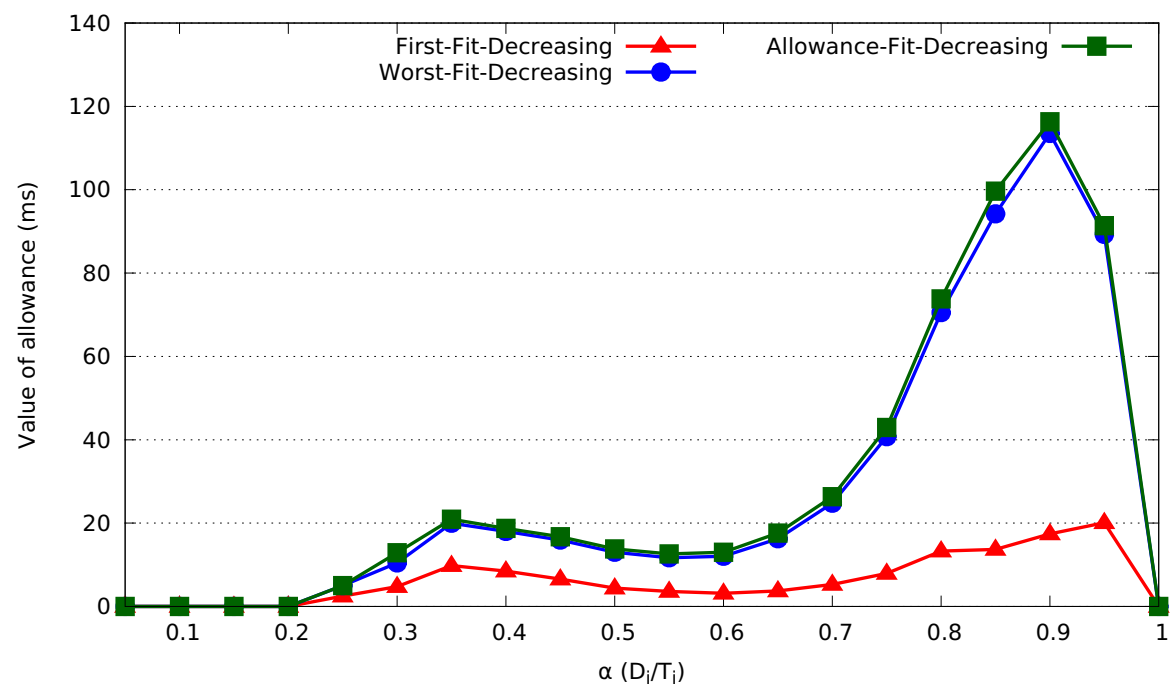

Fig. 3. Comparison of minimum allowance for the tasks partitioning heuristics on 4 processors

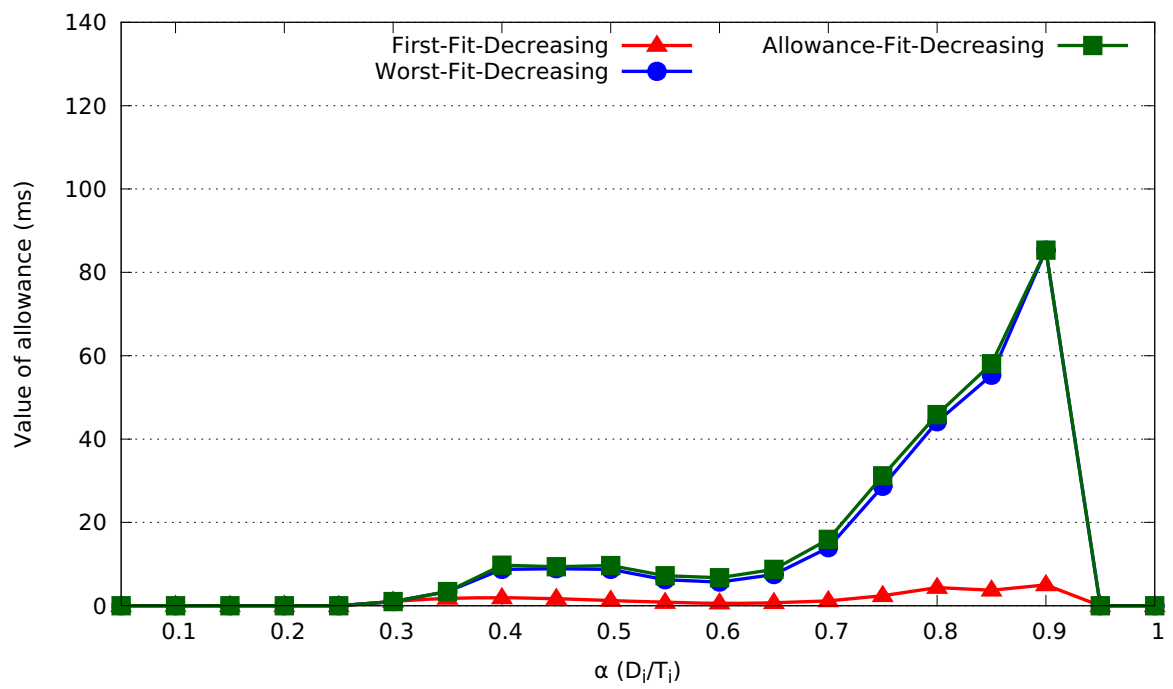

Fig. 4. Comparison of minimum allowance for the tasks partitioning heuristics on 8 processors

We show in Fig 5 the comparison between the computational time of the three heuristics. AFD offers better results than WFD in terms of minimum allowance. But the computation time of AFD is 6 to 10 greater than the computation time of WFD. For a robust allocation to the WCETs overruns, it is interesting to use AFD. But when tasks must be accepted online, WFD is a preferable choice. 


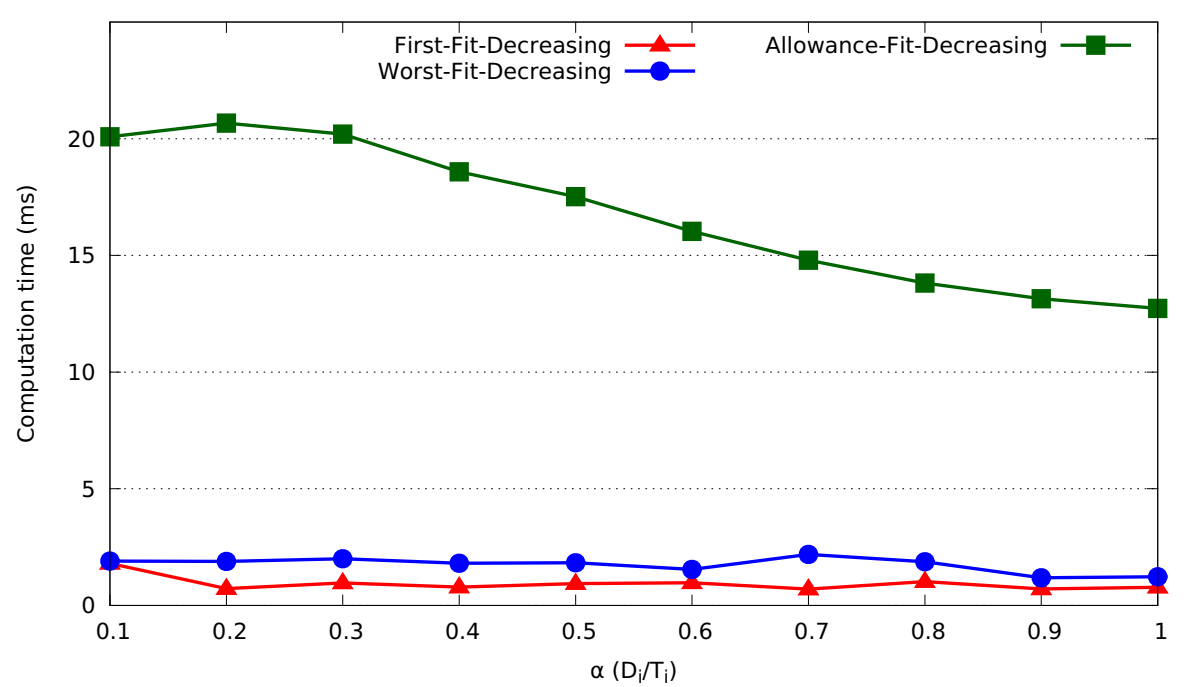

Fig. 5. Comparison of computation time of the heuristics

\section{Conclusion}

We have proposed a fixed-priority partitioned scheduling for homogeneous processors which maximizes the allowance of the execution duration. This scheduling is more robust than the others based on $\mathrm{FF}, \mathrm{BF}$ or NF because during allocation of the tasks, the processor offering the greatest value of allowance is chosen. In terms of maximization of allowance, Allowance-Fit is slighty efficient than Worst-Fit. But in terms of computation time, Worst-Fit is largelly better than Allowance-Fit. Thus Worst-Fit is a good heuristic to maximize the robustness of a partitioned system of real-time tasks. In a future work, we will extend this approach to the class of restricted migration scheduling to improve the schedulability of our solution. In such a scheduling, the different jobs of a recurring task can migrate from a processor to another, but no migration is allowed during the execution of the job.

\section{References}

1. Liu, C.L., Layland, J.W.: Scheduling algorithms for multiprogramming in a hard real-time environment. Journal of the ACM 20(1), 47-61 (1973)

2. Leung, J.Y.T., Whitehead, J.: On the complexity of fixed-priority scheduling of periodic, real-time tasks. Performance Evaluation 2(4), 237-250 (1982)

3. Davis, R.I., Burns, A.: Robust priority assignment for fixed priority real-time systems. In: Proceedings of the 28th Real-Time Systems Symposium (RTSS), Tucson, Arizona, USA, pp. 3-14. IEEE Computer Society, Los Alamitos (December 2007) 
4. Bertogna, M.: Evaluation of existing schedulability tests for global EDF. In: Proceedings of the 38th International Conference on Parallel Processing Workshops (ICPPW), Vienna, Austria, pp. 11-18. IEEE Computer Society, Los Alamitos (September 2009); First International Workshop on Real-time Systems on Multicore Platforms: Theory and Practice (XRTS)

5. Burchard, A., Liebeherr, J., Oh, Y., Son, S.H.: New strategies for assigning realtime tasks to multiprocessor systems. IEEE Transactions on Computers 44(12), 1429-1442 (1995)

6. Oh, Y., Son, S.H.: Allocating fixed-priority periodic tasks on multiprocessor systems. Real-Time Systems 9(3), 207-239 (1995)

7. Andersson, B., Jonsson, J.: Preemptive multiprocessor scheduling anomalies. In: Proceedings of the 16th International Parallel and Distributed Processing Symposium (IPDPS), Fort Lauderdale, Florida, USA, pp. 12-19. IEEE Computer Society, Los Alamitos (April 2002)

8. Fisher, N., Baruah, S.K., Baker, T.P.: The partitioned scheduling of sporadic tasks according to static-priorities. In: Proceedings of the 18th Euromicro Conference on Real-time Systems (ECRTS), Dresden, Germany, pp. 118-127. IEEE Computer Society, Los Alamitos (July 2006)

9. Lakshmanan, K., Rajkumar, R., Lehoczky, J.P.: Partitioned fixed-priority preemptive scheduling for multi-core processors. In: Proceedings of the 21st Euromicro Conference on Real-time Systems (ECRTS), Dublin, Ireland, pp. 239-248. IEEE Computer Society, Los Alamitos (July 2009)

10. Dibble, P.: Jsr 1: Real-time specification for java (December 1998)

11. Bougueroua, L., George, L., Midonnet, S.: Dealing with execution-overruns to improve the temporal robustness of real-time systems scheduled FP and EDF. In: Proceedings of the 2nd International Conference on Systems (ICONS), SainteLuce, Martinique, 8 p. IEEE Computer Society, Los Alamitos (April 2007)

12. Bini, E., Di Natale, M., Buttazzo, G.C.: Sensitivity analysis for fixed-priority realtime systems. In: Proceedings of the 18th Euromicro Conference on Real-time Systems (ECRTS), Dresden, Germany, pp. 13-22. IEEE Computer Society, Los Alamitos (April 2006)

13. Davis, R.I., Zabos, A., Burns, A.: Efficient exact schedulability tests for fixed priority real-time systems. IEEE Transactions on Computers 57(9), 1261-1276 (2008)

14. Garey, M.R., Johnson, D.S.: Computers and Intractability: A Guide to the Theory of NP-Completeness (1979)

15. Coffman Jr., E.G., Garey, M.R., Johnson, D.S.: Approximation algorithms for bin packing: A survey. In: Approximation Algorithms for NP-Hard Problems, pp. 4693. PWS Publishing Co., Boston (1996)

16. Audsley, N.C., Alan, B., Tindell, K.W., Wellings, A.J.: Applying new scheduling theory to static priority pre-emptive scheduling. Software Engineering Journal 8(5), 284-292 (1993)

17. Bini, E., Buttazzo, G.C.: Biasing effects in schedulability measures. In: Proceedings of the 16th Euromicro Conference on Real-time Systems (ECRTS), Catania, Sicily, Italy, pp. 196-203. IEEE Computer Society, Los Alamitos (June - July 2004) 